\title{
A rudimentary touch input device for visual displays
}

\author{
GEOFF CUMMING \\ La Trobe University, Bundoora, Australia 3083
}

\begin{abstract}
An inexpensive and simple device that can be fitted to existing computer visual display units and that gives a limited touch input capability is described. Small transparent acrylic pieces are held in front of the CRT by stiff wires attached to microswitches. The device has proved useful in the provision of computer-assisted instruction for young children.
\end{abstract}

Touching some chosen point on a visual display is a very appealing way of giving a message or command to a computer. The link between user and computer seems natural and direct, particularly when the computer responds promptly to the touch input. The naturalness is probably greatest when the user touches with a finger some chosen part of test or diagram material presented on a visual display unit (VDU), but touch input may alternatively be by light pen or stylus. A sensitive tablet may be touched, rather than the VDU surface itself.

Besides naturalness and immediacy, touch input offers two chief advantages. When dealing with complex spatial layouts, a touch device, particularly one with the accuracy of a fine stylus, allows points in the layout to be specified simply, precisely, and reliably. Some design and drafting systems exploit this feature of touch input.

Second, touch input can be used even by very young children, or others for whom a keyboard might be too complex. The PLATO computer-based education system, for example, makes heavy use of touch input, particularly in applications with young children (Obertino, Fillman, Gilfillian, \& Silver, 1977).

There are some drawbacks, however. Touch with a finger can give input of only quite limited precision, and repeated lifting of the arm and hand to touch the screen (if in normal vertical orientation) can be tiring. Besides, skilled use of a keyboard certainly gives very much higher data rates than any touch system. Design considerations for the use of touch in interactive computer systems are discussed by Shneiderman (1980).

Many technical approaches to the provision of touch input have been investigated, and several are now in use in commercially available systems. Carroll Manufacturing Corporation (1212 Hagan Street, Champaign, Illinois 61820) offers devices based on a grid of infrared beams just in front of the screen. Elographics, Inc. (1976 Oak Ridge Tumpike, Oak Ridge, Tennessee 37830), uses a thin, almost transparent membrane placed just in front of the display screen; a finger touch depresses the membrane, causing an electrical contact indicating the position touched. TSD Display Products, Inc. (35 Orville Drive, Bohemia, New York 11716), uses acoustic surface waves set up on the front surface of the display and reflected "radar-fashion" from a finger to locate the point touched.

Touch devices usually have to be built into the VDU at manufacture, or at least fitted as part of system packaging at the OEM stage. But manufacturers will supply prototyping kits for investigative use. The difficulty of fitting touch devices to existing VDUs and the moderately high cost (most over $\$ 1,000$ ) probably explain why touch devices are not yet widely available for hobby computers.

Many hobby computers do offer paddle or joystick controls, similar to those now familiar from video games, which link the moving of a knob or lever to the shifting of a visible cursor across the screen. A recent variant is the "mouse," a small hand-held object that is rolled around on a table or other flat surface to move the cursor around on the screen. The user can indicate a choice by moving the cursor to a selected point on the screen and then, perhaps, press an "enter" button to signal to the computer that the current cursor position should be taken as the chosen point. Such devices are cheap, widely available, and excellent for many applications. But they do not have the speed and directness of touch input, and so, in many applications, particularly with young children, they do not offer a fully adequate alternative to a touch device. This is, however, a rapidly developing field. Computer input devices other than keyboards are likely to increase markedly in power and ease of use.

The present paper describes a rudimentary touch device built for use in work on computer-assisted instruction (CAI) for beginning readers. The work follows that of Atkinson (1974): Children receive a sequence of very simple exercises with letters, words, parts of words, and, finally, sentences. In the original Atkinson work and in our first trials (Cumming \& Willox, 1982), the children made only typed responses. Even first-grade children coped surprisingly well with the standard keyboard, but the necessity to type all responses seemed to limit the type of task that could be asked of the children. Emphasis was inevitably on the spelling and internal structure of words, rather than holistically on broad shape or meaning. Availability of touch 
response as well as the type response mode would open up a broader range of kinds of exercise to the CAI designer.

After investigating the commercial options and costs and considering that we only needed touch to specify among a small group of response options, we built the device shown in Figure 1.

As can be seen from the figure, rectangles $(28 \mathrm{x}$ $45 \mathrm{~mm}$ ) of thin, clear acrylic sheet are positioned some $4.7 \mathrm{~mm}$ in front of the lower part of the VDU screen. These touch panels are glued to short lengths of stiff wire attached to microswitches mounted in a small box attached below the screen. Touching a panel pushes it forward, the screen surface itself forming a natural stop to the movement, and triggers the corresponding microswitch. The microswitches are connected in parallel with some chosen keys on the keyboard, in our case the digits $1-5$, so that touching a panel is equivalent to pressing the corresponding key. Programs can be arranged to recognize the touch and respond accordingly.

Details of the construction and the microswitches used are not given, since the geometry of the screen and its enclosure are the most strongly determining aspects for the design of the device and these will vary greatly for different VDUs. The panels and microswitches should be chosen and arranged so that a light touch is sufficient and gives a definite small click to confirm that the response has been registered. The spring return must be reliable and prompt.

In use, the cursor-addressing capability of the terminal is used to present the words or other items that are the response options squarely behind the touch panels. The items are clearly visible through the panels, and "touching the chosen item" is in practice a very natural and casy movement, which results in tripping of the microswitch.

We used the touch device for CAI in reading with first-grade children, including some with low reading levels (Cumming \& Willox, 1982). The children gave touch responses immediately and without any problem. Observation of some children using touch responses and other children giving typed responses confirmed that

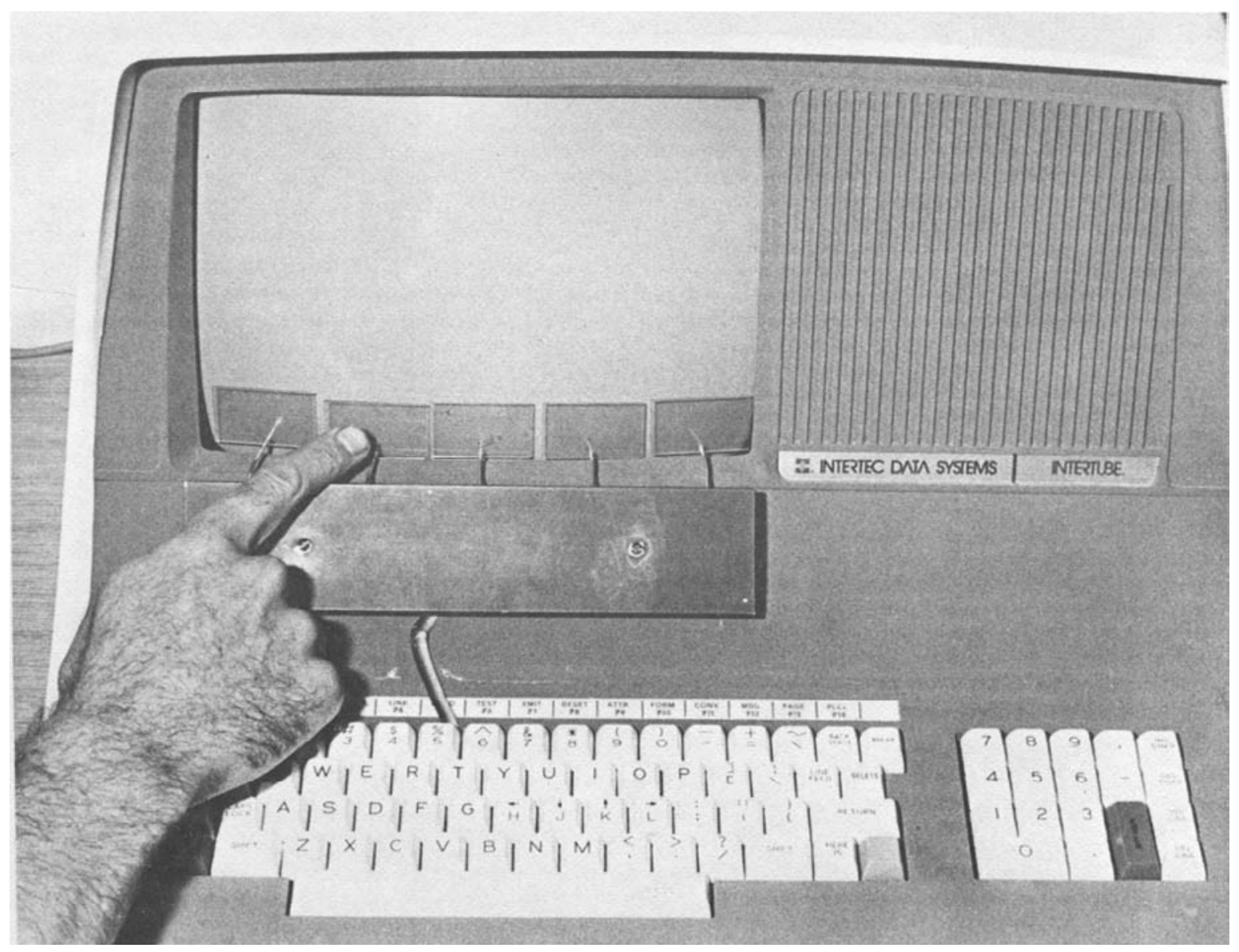

Figure 1. The VDU fitted with the touch device. The touch panels, each mounted on a length of stiff wire connected to a microswitch in the box, may be seen forming a line along the lower part of the screen. 
the two response modes did indeed seem to lead to dif fering treatment of items, and so, to be appropriate for different types of CAI exercises. Having both response modes available, even if touch is only in this primitive, restricted form, increases greatly the scope for CAI designers and should lead to more varied and interesting CAI, calling for a wider variety of cognitive skills on the part of the child.

One slight difficulty is related to one characteristic of the terminal: If a key is held down for more than a full $1 \mathrm{sec}$ or so, a stream of the corresponding character is emitted. So, if the child maintains touch pressure for several seconds, many characters are "seen" by the computer, rather than just one. Choice of an appropriate microswitch that gives a brief rather than continuous contact closure in response to sustained touching would solve the problem, but it is easier to ask children to give only a brief (less than $1-\mathrm{sec}$ ) touch. Children as young as 6 years have no difficulty achieving this.

Since deciding to build a touch device, we have had some informal experience with young children using a device based on the acoustic wave principle and another based on the infrared beam approach. The acoustic wave device sometimes gives false positive readings if dirty finger marks are made on the screen, a quite likely occurrence when working with young children. The infrared device requires that a single finger be used: Since the beams are inevitably a little in front of the screen, the device will sometimes erroneously pick up a dangling lower finger, and it can misplace even a single index finger if it is slanted too severely.

Our touch device is obviously rudimentary and limited, but it is quick and inexpensive to build, reliable, and well accepted by users. It bypasses problems that may arise with some other ways of providing touch input for young children. Besides its use with CAI, it may well be useful in applications with operating systems, or other complex packages in which it is desireable to keep several options for action demonstrably available to the user. Options such as NEXT, BACK, HELP, DELETE, and EXIT can be displayed through the touch panels whenever they are active.

\section{REFERENCES}

Atrinson, R. C. Teaching children to read using a computer. American Psychologist, 1974, 29, 169-178.

Cumming, G., \& Willox, N. Supplementary computer-assisted instruction for beginning readers: Australian trials. Australian Educational Researcher, 1982, 9, 31-45.

Obertino, P., Fillman, L., Gilfillian, J., \& Silver, D. Elementary reading on PLATO IV. Urbana-Champaign: University of Illinois, Computer-Based Educational Research Laboratory, 1977. (ERIC Document Reproduction Service No. ED 163993).

ShNeiderman, B. Software psychology: Human factors in computer and information systems. Cambridge, Mass: Winthrop, 1980.

(Received for publication August 27, 1982; revision accepted October 25, 1982.) 